\title{
CLASSICAL QUOTIENT RINGS OF GENERALIZED MATRIX RINGS
}

\author{
DAVID G. POOLE \\ Department of Mathematics, Trent University \\ Peterborough, Ontario, Canada, K9J 7B8 \\ e-mail: DPOOLE@TrentU.ca \\ and \\ PATRICK N. STEWART \\ Department of Mathematics, Statistics and Computing Science \\ Dalhousie University, Halifax, Nova Scotia, Canada, B3H 3J5 \\ e-mail: stewart@cs.dal.ca
}

(Received March 18, 1993)

\begin{abstract}
An associative ring $R$ with identity 1 is a generalized matrix ring with idempotent set $E$ if $E$ is a finite set of orthogonal idempotents of $R$ whose sum is 1 . We show that, in the presence of certain annihilator conditions, such a ring is semiprime right Goldie if and only if $e R e$ is semiprime right Goldie for all $e \in E$, and we calculate the classical right quotient ring of $R$.
\end{abstract}

KEY WORDS AND PHRASES. Generalized matrix ring, quotient ring, Goldie conditions. 1991 AMS SUBJECT CLASSIFICATION CODES. 16P60, 16 U20.

\section{INTRODUCTION.}

Each ring considered in this paper is associative and has an identity. Such a ring $R$ is a generalized matrix ring with idempotent set $E$ if $E$ is a finite set of orthogonal idempotents of $R$ whose sum is 1 .

In this paper, we show that, in the presence of certain non-degeneracy conditions, a generalized matrix ring $R$ with idempotent set $E$ is semiprime right Goldie if and only if $e R e$ is semiprime right Goldie for all $e \in E$, and we calculate the classical right quotient ring of $R$. Kerr's example [4] of a right Goldie ring whose matrix ring is not right Goldie shows that our semiprimeness condition cannot be omitted.

Examples of generalized matrix rings include incidence algebras of directed graphs with a finite number of vertices (see [5] and [9]), structural matrix rings (see Van Wyk [13] and subsequent papers), endomorphism rings of finite direct sums of modules and Morita context rings. Sands [10] observed that if $[S, V, W, T]$ is a Morita context, then

$$
\left[\begin{array}{cc}
S & V \\
W & T
\end{array}\right]
$$

is a ring. These Morita context rings are precisely generalized matrix rings with idempotent sets $E$ such that $|E|=2$, and they have been widely studied. In particular, we note Amitsur's paper [1], the survey paper [6], McConnell and Robson's treatment in ([7], 1.1 and 3.6) and 
Müller's computation of the maximal quotient ring in [8].

A generalized matrix ring $R$ with idempotent set $E$ is called a piecewise domain if for all $e, f, g \in E, x \in e R f$ and $y \in f R g$, we have $x y=0$ implies $x=0$ or $y=0$. These rings have been studied in some detail - see, for instance, [2] and [3].

We denote the prime radical of a ring $R$ by $p(R)$ and if $\epsilon$ and $f$ are idempotents of $R, \epsilon \neq f$, $p(e R f)$ denotes the set $\{x \in e R f: x f R e \subseteq p($ ( Rt) $\}$.

PROPOSITION 1.1 (Sands [10]). If $R$ is a generalized matrix ring with idempotent set $E$, then

$$
p(R)=\sum_{e, f \in E} p(e R f) .
$$

PROOF. If $|E|=1$ there is nothing to prove and if $|E|=2$ this is Theorem 1 in Sands [10]. Assume now that $|E|=n>2$ and that the theorem is true for generalized matrix rings with idempotent sets of cardinality less than $n$. Let $e \in E$ and set $E^{\prime}=\{e, 1-e\}$. Then $R$ is a generalized matrix ring with idempotent set $E^{\prime}$ and so Sands' result implies that

$$
p(R)=p(e R e)+p(e R(1-e))+p((1-e) R e)+p((1-e) R(1-e)) .
$$

Since $(1-e) R(1-e)$ is a generalized matrix ring with idempotent set $E_{1}=E \backslash\{e\}$, our induction hypothesis implies that

$$
p((1-e) R(1-e))=\sum_{f, g \in E_{1}} p(f R g) .
$$
Also, it is clear that $p(e R(1-e))=\sum_{f \in E_{1}} p(e R f)$ and $p((1-e) R e)=\sum_{f \in E_{1}} p(f R e)$, so the result
follows.

Let $R$ be a generalized matrix ring with idempotent set $E$. We say that the pair $(R, E)$ satisfies the left (respectively, right) annihilator condition if for all $e, f \in E, 0 \neq x \in e R f$ implies that $x f R e \neq 0$ (respectively, $f R e x \neq 0$ ). This concept is defined in [12] where right and left are interchanged.

COROLLARY 1.2. (Wauters and Jespers [12]). The following conditions on a generalized matrix ring with idempotent set $E$ are equivalent.

(a) $R$ is semiprime.

(b) $(R, E)$ satisfies the left annihilator condition and $e R e$ is semiprime for all $e \in E$.

(c) $(R, E)$ satisfies the right annihilator condition and $e R e$ is semiprime for all $e \in E$.

\section{THE GOLDIE CONDITIONS.}

The right singular ideal of a ring $S$ will be denoted by $Z(S)$, and the right singular submodule of a right $S$-module $M$ will be denoted by $Z(M)$. So, if $R$ is a generalized matrix ring with idempotent set $E$ and $e, f \in E$ with $e \neq f$, then $Z(e R e)$ is the right singular ideal of the ring $e R e$ and $Z(e R f)$ is the right singular submodule of the right $f R f$-module $e R f$.

PROPOSITION 2.1. Let $R$ be a generalized matrix ring with idempotent set $E$ and suppose that $(R, E)$ satisfies the left annihilator condition. Then

$$
Z(R)=\sum_{e, f \in E} Z(e R f)
$$

PROOF. Let $e, f \in E$ and suppose that $x \in Z(R)$. Then $\operatorname{exf} \in Z(R)$, so there is an essential right ideal $I$ of $R$ such that $\operatorname{exf} I=0$. To show that exf $\in Z(e R f)$ it suffices to show that $f I f$ is an essential right ideal of $f R f$. Let $A$ be a nonzero right ideal of $f R f$. Because $I$ is essential, 
$I \cap A R \neq 0$. Let $0 \neq u \in I \cap A R$. Since $I \cap A R$ is a right ideal there is an idempotent $g \in E$ such that $0 \neq u g \in I \cap A R$, and $u g=f u g$ because $u g \in A R \subseteq f R f R$. Since $(R, E)$ satisfies the left annihilator condition, $0 \neq(f u g) g R f \subseteq(I \cap A R) \cap f R f \subseteq f R f \cap A$. It follows that

$$
Z(R) \subseteq \sum_{\imath \in E} Z(e R f)
$$

Conversely, suppose that $\epsilon, f \in E$ and $y=\epsilon y f \in Z(\epsilon R f)$. Then $y H=0$ for some essential right ideal $H$ of $f R f$. Let $J=\{r \in R: f r \in H R\}$. Clearly, $J$ is a right ideal of $R$ and $y J=e y f J=(e y f) f J \subseteq(e y f) H R=0$, so to show that $y \in Z(R)$ it is enough to show that $J$ is essential in $R$. Let $B$ be a nonzero right ideal of $R$. If $f B=0$, then $B \subseteq J$ and so $B \cap J \neq 0$. Now assume $f B \neq 0$. Then $f B g \neq 0$ for some $g \in E$, and so the left annihilator condition implies that $f B f \neq 0$. So we see that $f B f$ is a nonzero right ideal of $f R f$. Thus $f B f \cap H \neq 0$ and so $B \cap J \neq 0$ because $H \subseteq H R$.

COROLLARY 2.2. If $R$ is a generalized matrix ring with idempotent set $E$ such that $(R, E)$ satisfies the left annihilator condition, then $R$ is nonsingular if and only if $e R e$ is nonsingular for all $e \in E$.

PROOF. In view of the proposition, we need only show that $Z(R) \neq 0$ implies that $Z(e R e) \neq 0$ for some $e \in E$. Suppose that $0 \neq x \in Z(R)$. Then $0 \neq e x f \in Z(R)$ for some $e, f \in E$. The right annihilator condition implies that $(e x f) f R e \neq 0$ and so $e R e \cap Z(R) \neq 0$. It now follows from the proposition that $Z(e R e) \neq 0$.

The right uniform dimension of a ring $R$ (respectively, right $R$-module $M$ ) will be denoted by $d(R)$ (respectively, $d(M)$ ).

PROPOSITION 2.3. Let $R$ be a generalized matrix ring with idempotent set $E$ such that $(R, E)$ satisfies the left annihilator condition. If $d(R)<\infty$ then $d(e R e)<\infty$ for all $e \in E$. Moreover, if $R$ is semiprime and $d(e R e)<\infty$ for all $e \in E$, then $d(e R f)<\infty$ for all $e, f \in E$ and hence $d(R)<\infty$.

PROOF. Assume that $d(R)<\infty, e \in E$ and $\sum A_{1}$ is a direct sum of nonzero right ideals of $e R e$. To prove that $d(e R e)<\infty$ it is enough to show that $\sum A_{\mathrm{t}} R$ is direct, and to accomplish this we need only show that $\sum A_{\mathbf{a}} R f$ is direct for each $f \in E$. Suppose that $f \in E$ and $b_{\mathbf{t}} \in A_{\mathbf{a}} R f$ are such that $\sum b_{1}=0$. Since $b_{\imath} f R e \subseteq A_{1}$ and $\sum A_{\imath}$ is direct, $b_{\imath} f R e=0$ for all $i$. Thus the left annihilator condition implies that $b_{\mathbf{i}}=0$ for all $i$ and hence $\sum A_{\mathbf{a}} R f$ is direct.

Now assume that $d(e R e)<\infty$ for all $e \in E$ and suppose that $\sum N_{\text {, }}$ is a direct sum of nonzero $f R f$-submodules of $e R f$. Since $0 \neq N_{1} \subseteq e R f$ the left annihilator condition implies that $N_{\imath} f R e \neq 0$, and each $N_{i} f R e$ is a right ideal of $e R e$. Let $K=N_{\jmath} f R e \cap \sum\left\{N_{\imath} f R e: i \neq j\right\}$. Then $K e R f \subseteq N_{j} \cap \sum\left\{N_{i}: i \neq j\right\}$ and so $K e R f=0$. Since $K^{2} \subseteq K e R f, K^{2}=0$ and so $K=0$ because $e R e$ is semiprime by Corollary 2. Thus $\sum N_{\mathfrak{t}} f R e$ is direct and so $d(e R f) \leq d(e R e)$. It follows that $R$ has finite right uniform dimension as a right $\left(\sum_{e \in E} e R e\right)$-module and so certainly $d(R)<\infty$ From Corollary 2, Corollary 4 and Proposition 5 we obtain the following theorem.

THEOREM 2.4. Let $R$ be a generalized matrix ring with idempotent set $E$. If $R$ is semiprime right Goldie, then so too are the rings $e R e, e \in E$. Conversely, if $(R, E)$ satisfies the left annihilator condition and $e R e$ is semiprime right Goldie for all $e \in E$, then $R$ is semiprime right Goldie.

\section{THE QUOTIENT RING.}

Let $S$ and $T$ be rings and let $M$ be an $S-T$-bimodule. We say that $M$ satisfies the right 
bimodule Ore condition if for each $m \in M$ and each regular clement $c \in S$ there is an $m_{1} \in M$ and a regular $c_{1} \in T$ such that $m c_{1}=c m_{1}$.

PROPOSITION 3.1. If $R$ is a semiprime right Goldie generalized matrix ring with idempotent set $E$. then $\epsilon R f$ satisfies the right bimodule Ore condition for all c. $f \in E$.

PROOF. Let $m \in \in R f$ and suppose that $c$ is regular in $\in R$. Define $\theta: c R f \rightarrow c c R f$ by $\theta(x)=c x$ for all $x \in e R f$. Clearly $\theta$ is an $f R f$-module homomorphism and we now check that $\theta$ is a monomorphism. Suppose that $x \in c R f$ and $c x=0$. Then $c x f R c=0$ which implies that $x f R \epsilon=0$ because $c$ is regular. But then the loft annililator condition implies that $x=0$ as required.

From Theorem 6 and Proposition 5 we know that $e R f$ has finite right uniform dimension as a right $f R f$-module. Since $e R f$ and $c e R f$ are isomorphic $f R f$-modules, $d(c e R f)=d(e R f)$ and so $c e R f$ is an essential $f R f$ submodule of $e R f$. Hence $\{y \in f R f: m y \in c e R f\}$ is an essential right ideal of $f R f$ which, since $f R f$ is semiprime right Goldie, must contain the required regular element $c_{1}$.

If $S$ is semiprime right Goldie, $Q(S)$ denotes the classical right quotient ring of $S$ and if $M$ is a right $S$-module, $Q(M)=M \otimes{ }_{S} Q(S)$. Using the right common denominator property of Ore sets we see that every element of $Q(M)$ is of the form $m \otimes c^{-1}$ where $m \in M$ and $c$ is regular in $S$. In what follows we shall write $m c^{-1}$ instead of $m \otimes c^{-1}$.

THEOREM 3.2. If $R$ is a semiprime right Goldie generalized matrix ring with idempotent set $E$, then

$$
Q(R)=\sum_{e, f \in E} Q(e R f)
$$

PROOF. For each $e \in E, \epsilon R e$ embeds in $Q(e R e)$ and we now check that for $e, f \in E, e \neq f, e R f$ embeds in $Q(e R f)$. Suppose that $c$ is regular in $f R f, x \in e R f$ and $x c=0$. Then $f \operatorname{Rexc}=0$ and so $f \operatorname{Rex}=0$ because $c$ is regular in $f R f$. Thus $\operatorname{exf} \operatorname{Rexf}=0$ and hence $0=e x f=x$ since $R$ is semiprime. This shows that $e R f$ is a torsion free $f R f$-module and so $e R f$ embeds in $Q(e R f)$.

Let $e, f, g \in E, x \in e R f, y \in f R g$ and suppose that $c$ is regular in $f R f$ and $d$ is regular in $g R g$. Define $\left(x c^{-1}\right)\left(y d^{-1}\right)=x y_{1} c_{1}^{-1} d^{-1}$ where $y_{1}$ and $c_{1}$ are obtained from the right bimodule Ore condition: $y c_{1}=c y_{1}$. It is straightforward to check that this multiplication is well-defined and that as a result $Q=\sum_{e \in E} Q(e R f)$ becomes a generalized matrix ring with idempotent set $E$.

We now show that $Q$ is semiprime. It follows from Theorem 6 that $e R e$ is semiprime right Goldie for all $e \in E$ and hence $Q(e R e)$ is semiprime for all $e \in E$. In view of Corollary 1.2, it suffices to show that $(Q, E)$ satisfies the right annihilator condition. Let $y d^{-1} \in Q(f R e)$ be such that $Q(e R f) y d^{-1}=0$. Then $(e R f)\left(y d^{-1}\right)=0$ and so $(e R f) y=0$. From Corollary 1.2 we see that $(R, E)$ satisfies the right annihilator condition and so $y=0$. Thus $(Q, E)$ satisfies the right annihilator condition and hence $Q$ is semiprime.

Let $e, f \in E, e \neq f$. From Proposition 2.3 we see that $e R f$ has finite uniform dimension as a right $f R f$-module and so $Q(e R f)$ has finite uniform dimension as a right $Q(f R f)$-module. Since $Q(f R f)$ is semisimple Artinian it follows that $Q(e R f)$ is an Artinian $Q(f R f)$-module, and hence $Q$ is right Artinian by an argument similar to ([7], 1.1.7). Since we have already seen that $Q$ is semiprime, $Q$ is a semisimple Artinian ring.

To complete the proof, we need only show that $R$ is a right order in $Q$. Let $x \in Q$, $x=\sum_{e, f \in E} x(e, f)$ where $x(e, f) \in Q(\epsilon R f)$ for all $e, f \in E$. Using the right common denominator 
bimodule Ore condition if for each $m \in M$ and each regular element $c \in S$ there is an $m_{1} \in M$ and a regular $c_{1} \in T$ such that $m c_{1}=c m_{1}$.

PROPOSITION 3.1. If $R$ is a semiprime right Goldie generalized matrix ring with idempotent set $E$, then $e R f$ satisfies the right bimodule Ore condition for all $e, f \in E$.

PROOF. Let $m \in e R f$ and suppose that $c$ is regular in $e R e$. Define $\theta: e R f \rightarrow c e R f$ by $\theta(x)=c x$ for all $x \in e R f$. Clearly $\theta$ is an $f R f$-module homomorphism and we now check that $\theta$ is a monomorphism. Suppose that $x \in e R f$ and $c x=0$. Then $c x f R e=0$ which implies that $x f R e=0$ because $c$ is regular. But then the left annihilator condition implies that $x=0$ as required.

From Theorem 6 and Proposition 5 we know that $e R f$ has finite right uniform dimension as a right $f R f$-module. Since $e R f$ and $c e R f$ are isomorphic $f R f$-modules, $d(c e R f)=d(e R f)$ and so $c e R f$ is an essential $f R f$ submodule of $e R f$. Hence $\{y \in f R f: m y \in c e R f\}$ is an essential right ideal of $f R f$ which, since $f R f$ is semiprime right Goldie, must contain the required regular element $c_{1}$.

If $S$ is semiprime right Goldie, $Q(S)$ denotes the classical right quotient ring of $S$ and if $M$ is a right $S$-module, $Q(M)=M \otimes{ }_{S} Q(S)$. Using the right common denominator property of Ore sets we see that every element of $Q(M)$ is of the form $m \otimes c^{-1}$ where $m \in M$ and $c$ is regular in $S$. In what follows we shall write $m c^{-1}$ instead of $m \otimes c^{-1}$.

THEOREM 3.2. If $R$ is a semiprime right Goldie generalized matrix ring with idempotent set $E$, then

$$
Q(R)=\sum_{e, f \in E} Q(e R f)
$$

PROOF. For each $e \in E, e R e$ embeds in $Q(e R e)$ and we now check that for $e, f \in E, e \neq f, e R f$ embeds in $Q(e R f)$. Suppose that $c$ is regular in $f R f, x \in e R f$ and $x c=0$.

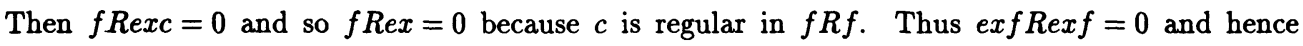
$0=e x f=x$ since $R$ is semiprime. This shows that $e R f$ is a torsion free $f R f$-module and so $e R f$ embeds in $Q(e R f)$.

Let $e, f, g \in E, x \in e R f, y \in f R g$ and suppose that $c$ is regular in $f R f$ and $d$ is regular in $g R g$. Define $\left(x c^{-1}\right)\left(y d^{-1}\right)=x y_{1} c_{1}^{-1} d^{-1}$ where $y_{1}$ and $c_{1}$ are obtained from the right bimodule Ore condition: $y c_{1}=c y_{1}$. It is straightforward to check that this multiplication is well-defined and that as a result $Q=\sum_{e \in E} Q(e R f)$ becomes a generalized matrix ring with idempotent set $E$.

We now show that $Q$ is semiprime. It follows from Theorem 6 that $e R e$ is semiprime right Goldie for all $e \in E$ and hence $Q(e R e)$ is semiprime for all $e \in E$. In view of Corollary 1.2, it suffices to show that $(Q, E)$ satisfies the right annihilator condition. Let $y d^{-1} \in Q(f R e)$ be such that $Q(e R f) y d^{-1}=0$. Then $(e R f)\left(y d^{-1}\right)=0$ and so $(e R f) y=0$. From Corollary 1.2 we see that $(R, E)$ satisfies the right annihilator condition and so $y=0$. Thus $(Q, E)$ satisfies the right annihilator condition and hence $Q$ is semiprime.

Let $e, f \in E, e \neq f$. From Proposition 2.3 we see that $e R f$ has finite uniform dimension as a right $f R f$-module and so $Q(e R f)$ has finite uniform dimension as a right $Q(f R f)$-module. Since $Q(f R f)$ is semisimple Artinian it follows that $Q(e R f)$ is an Artinian $Q(f R f)$-module, and hence $Q$ is right Artinian by an argument similar to ([7], 1.1.7). Since we have already seen that $Q$ is semiprime, $Q$ is a semisimple Artinian ring.

To complete the proof, we need only show that $R$ is a right order in $Q$. Let $x \in Q$, $x=\sum_{e, f \in E} x(e, f)$ where $x(e, f) \in Q(e R f)$ for all $e, f \in E$. Using the right common denominator 
property we can find, for each $f \in E$, an $a_{f} \in f R f$ and elements $y(e, f) \in e R f$ such that for all $e \in E, x(e, f)=y(e, f) a_{f}^{-1}$. Let $y=\sum_{e, f \in E} y(e, f)$ and $z=\sum_{f \in E} a_{f}$. Then $x=y z^{-1}$ and so $R$ is a right order in $Q$.

Let $R$ be a semiprime right Goldie ring with idempotent $e$. Clearly, $R$ is a generalized matrix ring with idempotent set $E=\{e, 1-e\}$ and so it follows from Theorem 2.4 that $e R e$ is semiprime right Goldie. This result seems to have been well-known for some time. Also, it follows from Theorem 3.2 that the classical right quotient ring of $e R e$ is $e Q e$ where $Q$ is the classical right quotient ring of $R$. This result is due to Small [11].

ACKNOWLEDGEMENT. This paper was written while the first author was on sabbatical at Dalhousie University. He would like to thank the Department of Mathematics, Statistics and Computing Science for its warm hospitality.

An earlier version of this paper included an unnecessary faithfulness assumption in Theorem 2.4. The authors are grateful to Michael V. Clase who supplied them with a preprint which lead to the removal of that assumption.

\section{REFERENCES}

1. AMITSUR, S.A., Rings of quotients and Morita contexts, J. Algebra 17 (1971), 273-298.

2. GORDON, R. and SMALL, L.W., Piecewise domains, J. Algebra 23 (1972), 553-564.

3. GORDON, R., Classical quotient rings of PWD's, Proc. Amer. Math. Soc. 36 (1972), 39-46.

4. KERR, J.W., An example of a Goldie ring whose matrix ring is not Goldie, J. Algebra 6 (1979), 590-592.

5. LEROUX, P. and SARRAILLÉ, J., Structure of incidence algebras of graphs, Comm. Algebra 9 (1981), 1479-1517.

6. LOUSTAUNAU, P. and SHAPIRO, J., Morita contexts, Noncommutative Ring Theory (Athens, OH, 1989), 80-992, Lecture Notes in Math., 1448, Springer, Berlin, 1990.

7. McCONNELL, J.C. and ROBSON, J.C., Noncommutative Noetherian Rings, Pure and Applied Mathematics, Wiley, New York, 1987.

8. MÜLLER, M., Rings os quotients of generalized matrix rings, Comm. Algebra 15 (1987), 1991-2015.

9. RIBENBOIM, P., The algebra of functions of a graph, Studia Sci. Math. Hung. 17 (1982), 120.

10. SANDS, A.D., Radicals and Morita contexts, J. Algebra 24 (1973), 335-345.

11. SMALL, L.W., Orders in Artinian rings II, J. Algebra 9 (1968), 266-273.

12. WAUTERS, P. and JESPERS, E., Rings graded by an inverse semigroup with finitely many idempotents, Houston J. of Math. 15 (1989), 291-304.

13. VAN WYK, L., Maximal left ideals in structural matrix rings, Comm. Algebra 16 (1988), 399-419. 


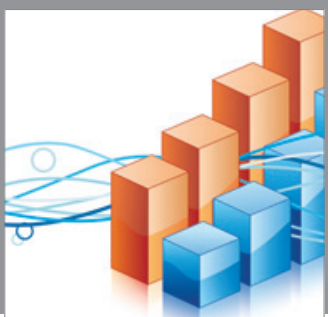

Advances in

Operations Research

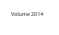

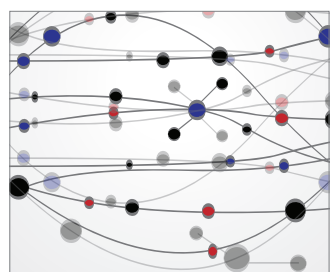

\section{The Scientific} World Journal
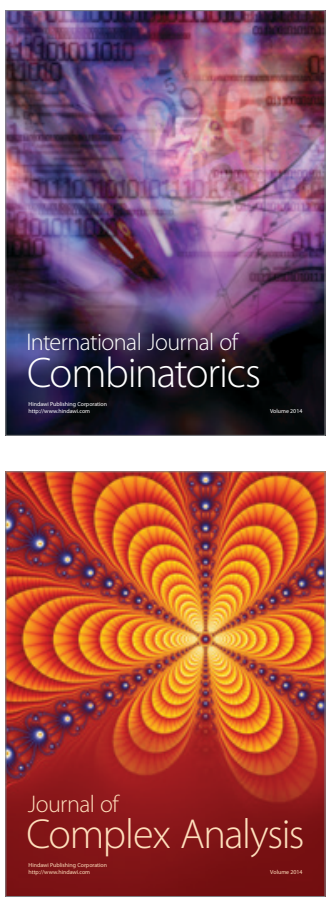

International Journal of

Mathematics and

Mathematical

Sciences
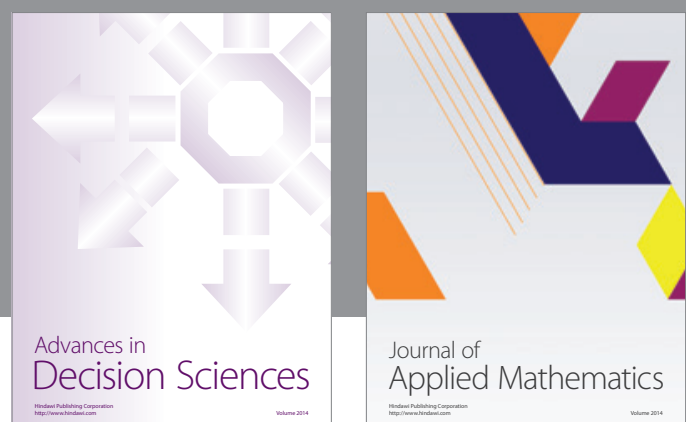

Journal of

Applied Mathematics
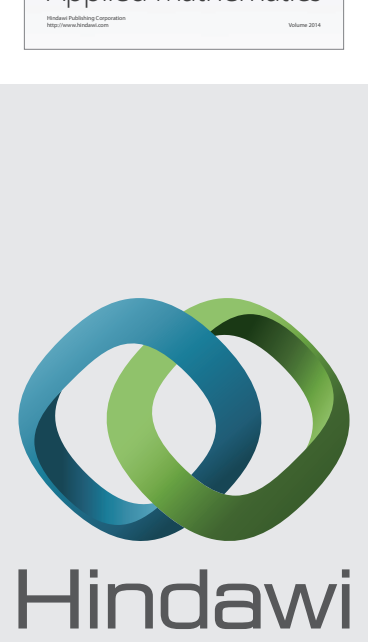

Submit your manuscripts at http://www.hindawi.com
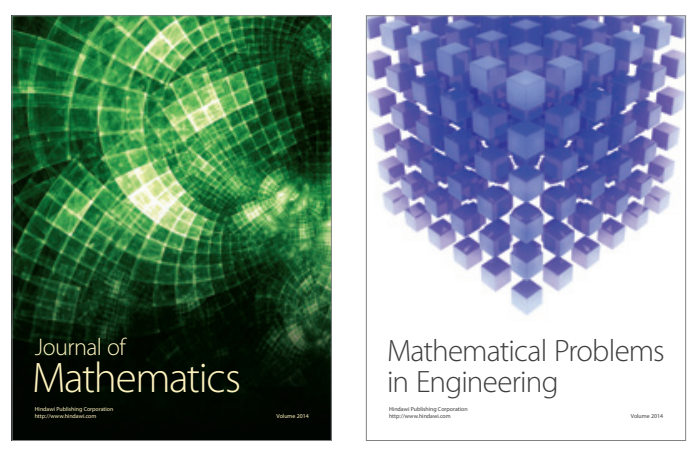

Mathematical Problems in Engineering
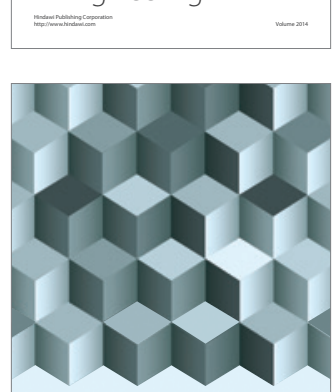

Journal of

Function Spaces
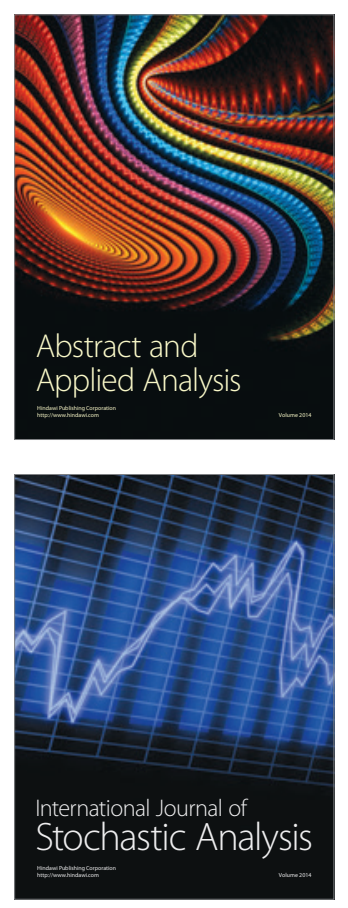

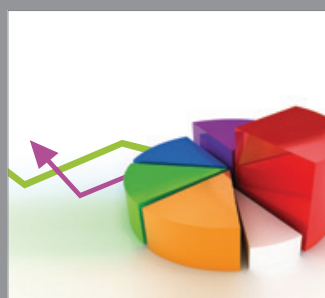

ournal of

Probability and Statistics

Promensencen
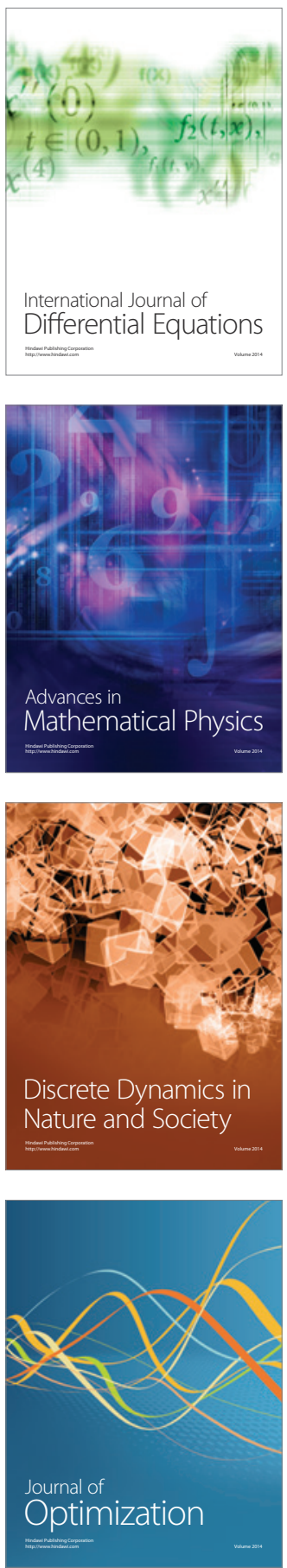